\title{
Acoustic measurement of the low-energy excitations in $\mathrm{Nd}_{2-x} \mathrm{Ce}_{x} \mathrm{CuO}_{4+\delta}$
}

\author{
F.Cordero, ${ }^{1}$ C. R. Grandini, ${ }^{2}$ and M. Ferretti ${ }^{3}$ \\ ${ }^{1}$ CNR, Istituto di Acustica "O.M. Corbino", Via del Fosso del Cavaliere 100, \\ I-00133 Roma, Italy and Unità INFM-Roma1, P.le A. Moro 2,I-00185 Roma,Italy \\ ${ }^{2}$ Universidade Estadual Paulista, Departamento de Fisica, 17.033-360,Bauru, SP, Brazil \\ ${ }^{3}$ Università di Genova, Dipartimento di Chimica e Chimica Industriale, \\ and Unità INFM-Genova, Via Dodecanneso 31, I-16146 Genova, Italy
}

The complex dynamic Young's modulus of ceramic $\mathrm{Nd}_{2-x} \mathrm{Ce}_{x} \mathrm{CuO}_{4}$ with $x=0,0.05$ and 0.20 has been measured from 1.5 to $100 \mathrm{~K}$ at frequencies of $1-10 \mathrm{kHz}$. In the undoped sample the modulus starts decreasing below $\sim 20 \mathrm{~K}$, instead of approaching a constant value as in a normal solid. The modulus minimum has been interpreted in terms of paraelastic contribution from the relaxation of the $\mathrm{Nd}^{3+} 4 f$ electrons between the levels of the ground state doublet, which is split by the interaction with the antiferromagnetically ordered $\mathrm{Cu}$ sublattice. The value of the splitting is found to be $0.34 \mathrm{meV}$, in excellent agreement with inelastic neutron scattering, infrared and specific heat experiments. With doping, the anomaly shifts to lower temperature and decreases in amplitude, consistently with a reduction of the local field from the $\mathrm{Cu}$ sublattice. 


\section{INTRODUCTION}

The crystal-field (CF) excitations of the $\mathrm{Nd}^{3+} 4 f$ electrons in $\mathrm{Nd}_{2-x} \mathrm{Ce}_{x} \mathrm{CuO}_{4}$ have been extensivelystudied, mainly by inelastic neutron scattering (INS) 14 and infrared spectroscopies.5 Although discrepancies exist between the proposed sets of phenomenological parameters in the CF hamiltonian, there is general agreement on the level scheme. 5 . The ground state multiplet of $\mathrm{Nd}^{3+}$ has $J=9 / 2$ and is split into five doublets, the separation of the first two being $14 \mathrm{meV}$; each doublet, in turn, is split by the interaction of the Nd magnetic moment with the local magnetic field, mainly created by the antiferromagnetic ordering of the $\mathrm{Cu}$ magnetic moments below $T_{\mathrm{N}}^{\mathrm{Cu}} \simeq 260 \mathrm{~K}$. The magnetic separation of the first doublet has been determined by INS, and passes from $0.39 \mathrm{meV}$ for the undoped case to $0.2 \mathrm{meV}$ for $x=0.15$.6 Such a low-energy excitation also appears as a $\mathrm{S}$ hottky anomaly in the low-temperature specific heat, 610 with values of the level splitting in agreement with the INS results; in principle, it should also give rise to an anomaly in the elastic moduli, if the level separation can be modulated by strain. To our knowledge, only two experiments have been reported on the astic properties of NCCO at liquid He temperatures, 11. 12 but no attempts have been made to relate those data to the elastic response of the ground state doublet. We made anelastic spectroscopy measurements in the $\mathrm{kHz}$ range on $\mathrm{Nd}_{2-x} \mathrm{Ce}_{x} \mathrm{CuO}_{4}$ with $x=0,0.05$ and 0.20 , and found a decrease of the Young's modulus at liquid He temperatures, which can be identified with the paraelastic response of the ground state doublet of $\mathrm{Nd}^{3+}$.

\section{EXPERIMENTAL}

The samples were prepared by solid state reaction, with repeated sintering and grinding of the powders up to obtaining X-ray diffraction spectra free of unwanted peaks; the methods used, together with a thorough thermodynamic study of the phase diagram and stability of the Nd-Ce-Cu-O system, are reported in Ref. 13. Generally, a reducing treatment is made on $\mathrm{Nd}_{2-x} \mathrm{Ce}_{x} \mathrm{CuO}_{4}$, in order to make it superconducting; the data presented here refer to the as-prepared or reoxygenated status.

The samples were cut as bars approximately $40 \times 4 \times$ $0.6 \mathrm{~mm}^{3}$. The dynamic Young's modulus, $E(\omega, T)=$ $E^{\prime}+i E^{\prime \prime}$, was measured by suspending the bars on their nodal lines on thin wires and electrostatically exciting their flexural vibrations; the 1st and 5th flexural modes were chosen, whose resonance frequencies $\omega / 2 \pi$ are approximately in the ratio $1: 13.3$. The vibration amplitude was measured by a frequency modulation technique, where the capacity between the sample and the electrode is inserted into a high frequency oscillator, and the resulting signal, after passing through an amplitudemodulation discriminator, is sent to a lock-in amplifier.
The real part $E^{\prime}$ of the Young's modulus is given by $E=\rho\left(0.975 \frac{\omega}{2 \pi} \frac{l^{2}}{h}\right)^{2}$, where $\rho, l$ and $h$ are the sample density, length and thickness; a correction for the porosity should be necessary, but we are only interested in the relative variation of $E$ with temperature. The elastic energy loss coefficient $Q^{-1}=E^{\prime \prime} / E^{\prime}$ was measured from the free decay of the vibration amplitude after switching off the excitation signal.

\section{RESULTS}

Figure 1 presents the complex Young's modulus of $\mathrm{Nd}_{2-x} \mathrm{Ce}_{x} \mathrm{CuO}_{4}$ at three doping levels, between 1 and $100 \mathrm{~K}$; only the data of the first mode are presented, since those at higher frequency are identical. The elastic energy loss coefficient decreases monotonously with temperature and with increasing doping, without any particular feature except for a small peak at $15 \mathrm{~K}$ for the undoped sample. Such a peak disappears after annealing in vacuum at high temperature, and, although several reorientational transitions of the $\mathrm{Nd}$ and $\mathrm{Cu}$ spins have beep served at low temperature with various techniques, $6,14,15$ it does not seem to be associated to any of these magnetic transitions. We will not discuss this peak further. The elastic modulus presents a nearly linear stiffening on cooling below room temperature, which levels off below $30 \mathrm{~K}$; contrary to a normal solid, however, the modulus does not stabilize on further cooling, but drops of several parts in $10^{3}$. The drop actually saturates at the lowest temperatures reached in the experiments (see also Fig. 2), and in some cases we measured the beginning of a rise below $1.5 \mathrm{~K}$. The temperature and amplitude of the drop decrease with increasing doping similarly to the Schottky anomaly in the specific heat, and therefore this feature might well be connected with the ground state doublet of the $\mathrm{Nd}^{3+}$ ions.

\section{DISCUSSION}

Most of the specific heat data below $10 \mathrm{~K}$ have been interpreted in terms of a Schgttky anomaly arising from the ground state doublet 610 with a splitting $\Delta(T)=$ $2 \mu_{0} B_{\mathrm{Cu}}(T)$ determined through $\mathrm{Nd}-\mathrm{Cu}$ exchange by the staggered magnetization $B_{\mathrm{Cu}}(T)$ of the $\mathrm{AF} \mathrm{Cu}$ sublattice below $T_{\mathrm{N}}^{\mathrm{Cu}}$; since $T_{\mathrm{N}}^{\mathrm{Cu}} \simeq 260 \mathrm{~K}, B_{\mathrm{Cu}}$ and the splitting $\Delta$ are practically constant below $50 \mathrm{~K}$. The existence of two levels $E_{i}$ separated by $\Delta$, whose internal energy is $U=$ $(\Delta / 2) \tanh \left(\Delta / 2 k_{\mathrm{B}} T\right)$, contributes to the molar specific heat with the Schottky term

$$
\delta C_{p}=\frac{\partial U}{\partial T}=k_{\mathrm{B}} c\left(\frac{\Delta}{2 k_{\mathrm{B}} T}\right)^{2} \frac{1}{\cosh ^{2}\left(\Delta / 2 k_{\mathrm{B}} T\right)},
$$

where $c$ is the molar concentration of the relaxing entities, in the present case the concentration $1-x / 2$ of $\mathrm{Nd}$ ions. 
On the other hand, if the splitting can pe modulated by a strain $\varepsilon$, paraelastic and diaelastic 617 contributions to the elastic modulus $M=\partial^{2} U / \partial \varepsilon^{2}$ are expected. Here we omit the tensorial character of the elastic properties, since we are dealing with the Young's modulus of polycrystalline samples, which contains a combination of several elastic constants and we are not entering in the details of the effect of the strain symmetry on the $\mathrm{Nd}^{3+}$ multiplets; then $M$ is identified with the Young's modulus $E$ and $\varepsilon$ is the corresponding effective strain.

The paraelastic contribution can be written a, 19,17 $\delta M_{\text {para }}=\sum_{i j} \frac{\partial n_{i}}{\partial E_{j}} \frac{\partial E_{i}}{\partial \varepsilon} \frac{\partial E_{j}}{\partial \varepsilon}$ and is due to the relaxation of the populations $n_{i}$, perturbed by the vibration strain $\varepsilon$ through the modulation of the energies $E_{i}$. For only two levels $E_{2}-E_{1}=\Delta$, one obtains 17

$$
\delta M_{\text {para }}=-\frac{c}{v_{0}} \gamma^{2} \frac{1}{4 k_{\mathrm{B}} T \cosh ^{2}\left(\Delta / 2 k_{\mathrm{B}} T\right)},
$$

where $v_{0}$ is the molecular volume and $\gamma=\partial \Delta / \partial \varepsilon$ is the deformation potential. Such a relaxation occurs with a characteristic rate $\tau^{-1}$, and therefore the dynamic modulus 18 has to be multiplied by a factor $(1+i \omega \tau)^{-1}$. The resulting real part of the dynamic modulus $M^{\prime}(\omega, T)$ acquires a frequency-dependent dispersion, while the imaginary part produces absorption in correspondence with the modulus dispersion. The present data, however, do not exhibit any frequency dependence of $M^{\prime}(\omega, T)$ or absorption peak, and this implies $\omega \tau \simeq 0$, namely the rate $\tau^{-1}$ is much faster than the measuring frequencies $\omega \sim 10^{4}-10^{5} \mathrm{~s}^{-1}$. Indeed, the $\mathrm{Cu}$ and $\mathrm{Nd}$ spip rates deduced from muon spin relaxation experiments 19 in samples with $x=0.20$ are faster than $10^{10} \mathrm{~s}^{-1}$.

If the first derivative $\gamma$ of the energy split with respect to strain is null for symmetry reasons, than one remains with the diaelastic term, $\delta M^{\text {dia }}=\frac{c}{v_{0}} \sum_{i} n_{i} \frac{\partial^{2} E_{i}}{\partial \varepsilon^{2}}$, which contains the second derivatives. The physical meaning of such a term is simply that the curvature of each energy level versus strain contributes to the corresponding elastic constant, and its contribution is weighted with the level population. For only two levels one obtains: 17

$$
\delta M_{\mathrm{dia}}=\frac{c}{v_{0}} \frac{1}{2} \frac{\partial^{2} \Delta}{\partial \varepsilon^{2}} \tanh \left(\frac{\Delta}{2 k_{\mathrm{B}} T}\right) .
$$

The continuous curves in Fig. 2 are fits with $\delta M_{\text {para }}$ given by Eq. 2 and assuming that the background modulus depends on temperature like an insulating Debye solid,20 $M(T)=M_{0}\left(1-a T^{4}\right)$. An additional term quadratic in $T$ is expected when the system becomes metallic, due to the energy of the free electrons, which increases quadratically with $T$; such a term, however, could be of importance only for the highest doping $x=0.20$, and still at $x=0.15$ some elastic constants have been found to be-well described by the quartic term alone below $60 \mathrm{~K} .22$ From the data at $x=0$ and 0.05 we find that $M(T)$ is described by the coefficient $a=-2.1 \times 10^{-10} \mathrm{~K}^{-4}$. The values of the splitting deduced from the fits of Fig. 2 are $\Delta / k_{\mathrm{B}}=4.0,2.25$ and
$1.3 \mathrm{~K}$ at $x=0,0.05$ and 0.20 respectively, while the amplitudes of the paraelastic contributions are in the ratios $1: 0.6: 0.05$. The splitting found for the undoped case is in excellent agreement with the values deduced from INS2 $\left(\Delta / k_{\mathrm{B}}=406 \mathrm{~K}\right)$, infrared spectroscopye $(3.8 \mathrm{~K})$ and specific heat $\left(\Delta / k_{\mathrm{B}}=4.5 \mathrm{~K}\right)$. Doping by Ce substitution introduces electrons mainly of $\mathrm{Cu} 3 d$ character, which cancel the magnetic moments of those $\mathrm{Cu}$ ions and therefore reduce the $\mathrm{Cu}_{-} \mathrm{Nd}$ exchange responsible for the ground state splitting. 9 As a result, the Schottky anomaly in the specific heat has been found to shift to lower temperature to broaden and to decrease in intensity with doping. trend, but the reduction in intensity is more marked than in the specific heat measurements; 6 possibly because of a reduction of the strain dependence of the splitting with doping.

At $x>0.15$ there is no long range AF order of the $\mathrm{Cu}$ spins, and the magnetic ground state splitting should disappear; nonetheless, the specific heat presents a peak up to $x=0.20 .10$ Our data at $x=0.20$ also present a residue due to the doublet with reduced splitting, but the value deduced from the fit, $\Delta / k_{\mathrm{B}}=1.2 \mathrm{~K}$, should be considered with caution in view of the possible influence of an electronic quadratic term in the temperature dependence of the modulus.

In some cases the fits to the specific heat data have been improved by assuming an additional temperature dependence of the magnetic splitting attributed to the ordering of the Nd momenta 6 which however affects the splitting by less than $10 \%$, or by assuming a three-level structure instead of a doublet.8 We chose to not overinterpret our data with additional parameters besides a simple Schottky anomaly with constant splitting below $50 \mathrm{~K}$ and the $T^{4}$ dependence of the background elastic modulus. In fact, the contribution of the doublet to the elastic response, Eq. 2, has a slower temperature dependence than the contribution to the specific heat, Eq. (1), in the high temperature limit, being proportional to $T^{-1}$ instead of $T^{-2}$; therefore, the elastic anomaly is spread to higher temperature where additional effects become important. In addition, the lowest temperature attained in our experiments was not sufficient to complete the lowtemperature side of the curves, so that some uncertainty in the determination of the minimum of $\delta M_{\text {para }}(T)$ exists. This means that, while it is easy to improve the fit by adding a temperature dependence of the splitting, by taking into account the contribution of the excited doublets, and adopting more sophisticated expressions for $M(T)$, it is difficult to distinguish which of the improvements is the important one. Still, the data presented here, together with other results on reduced samples, are sufficient to indicate the presence of a minimum in the modulus due to a paraelastic response, rather than a step-like anomaly due to a diaelastic response. Fits to the dialeastic expression, Eq. 3, are less satisfactory and yield values of $\Delta$ about twice larger than those of Fig. 2 , in contrast with the literature values. If both the di- 
aelastic and paraelastic terms are considered, with both $\gamma$ and $\partial^{2} \Delta / \partial \varepsilon^{2}$ as free parameters, the splitting $\Delta$ remains almost the same as reported here and up to half of the anomaly can be accounted for by the diaelastic term, without a significant improvement of the fit. We conclude that the anelastic response in the modulus is mainly due to the linear part of the response of the ground-state splitting to strain. The magnitude of the deformation potential $\gamma$ can be extracted inserting in Eq. 2 the value obtained from the fit, $\left(\delta M_{\text {para }} / M\right)_{\max }=0.0044$ at $2 \mathrm{~K}$ for $x=0$, and using $M=100 \mathrm{GPa}$, as determined from the resonance frequency (without corrections for the porosity); it turns out $\gamma=0.013 \mathrm{eV}$ for $x=0$ and $0.01 \mathrm{eV}$ for $x=0.05$.

As noted above, the absence of a rise of the absorption in correspondence to the decrease of the modulus implies that the relaxation rate for the $\mathrm{Nd}^{3+}$ spins to redistribute themselves within the ground states doublet is much faster than our measuring frequency, $\omega \sim$ $10^{4}-10^{5} \mathrm{~s}^{-1}$. Also, there are no signs of freezing of the $\mathrm{Cu}$ spins into a spin-glass or cluster glass states, which instead is observed in the hole-doped $\mathrm{CuO}_{2}$ planes of $\mathrm{La}_{2-x} \mathrm{Sr}_{x} \mathrm{CuO}_{4}$ and $\mathrm{YBa}_{2} \mathrm{Cu}_{3} \mathrm{O}_{6+x}, 21$ in $\mathrm{La}_{2-x} \mathrm{Sr}_{x} \mathrm{CuO}_{4}$ the frozen spin domins produce a distinct rise of the acoustic absorption,22 which is totally absent here. Similar conclusiens have been drawn from $\mu \mathrm{SR}$ relaxation experiments.19

To our knowledge, only two other experiments12,11 exist on the acoustic properties of $\mathrm{Nd}_{2-x} \mathrm{Ce}_{x} \mathrm{CuO}_{4}$ at liquid He temperatures, which however cannot be compared with the present one in a straightforward way; in fact, they report some elastic constants of single crystals, while we measured the Young's modulus of polycrystals, which contain a combination of all the compliances. 18 Fill et al. 11 found several anomalies in the elastic constants of undoped $\mathrm{Nd}_{2} \mathrm{CuO}_{4}$ as a function of temperature and magnetic field. In particular, the $c_{66}$ shear presented a minimum at $5 \mathrm{~K}$ with an amplitude nearly ten times larger than our minimum, and with a narrower shape, followed by a smaller decrease below $1 \mathrm{~K}$ tentatively attributed to a Schottky anomaly or magnetic ordering of $\mathrm{Nd}$ ions. Around $5 \mathrm{~K}$ also step-like changes have been found in other elastic constants, which have been interpreted as a ferrpelastic transition driven by an ordering of the Nd spins.11, 15 We cannot find a clear correspondence between those data and the ones presented here.

Regarding the doped material, Saint-Paul et al.12 measured the sound velocity of a single crystal of $\mathrm{Nd}_{1.85} \mathrm{Ce}_{0.15} \mathrm{CuO}_{4}$; the shear mode in the $a b$ plane did not present any anomaly down to $10 \mathrm{~K}$, but this is not in contrast with our data at $x=0.20$, where the decrease of the modulus is hardly detectable above $10 \mathrm{~K}$. Instead, the $c_{33}$ mode exhibited an upward deviation below $10 \mathrm{~K}$ with respect to the $-T^{4}$ dependence, opposite to our measurements. A possible explanation for the discrepancy is that the major contribution to the $c_{33}$ mode is diaelastic with positive $\partial^{2} \Delta / \partial \varepsilon^{2}$, while the Young's modulus of the polycrystal contains a predominant paraelastic contribution which cancels the diaelastic one.

\section{CONCLUSION}

The Young's modulus and elastic energy loss coefficient of $\mathrm{Nd}_{2-x} \mathrm{Ce}_{x} \mathrm{CuO}_{4}$ with $x=0,0.05$ and 0.20 have been measured from 1.5 to $100 \mathrm{~K}$. On approaching the lowest temperature, the modulus presents a drop whose temperature and amplitude decrease with increasing doping. The data have been interpreted in terms of paraelastic contribution from the relaxation of the $\mathrm{Nd}^{3+} 4 f$ electrons between the levels of the ground state doublet, which is split by the interaction with the antiferromagnetically ordered $\mathrm{Cu}^{2+}$ ions. Excellent agreement is found with the value of the splitting at zero doping deduced from INS, infrared and specific heat measurements, and the shift of the anomaly to lower temperature with doping is consistent with a reduction of the local field from the $\mathrm{Cu}$ sublattice. An effective deformation potential $\gamma=0.013 \mathrm{eV}$ is found for the strain derivative of the splitting at $x=0$, which decreases with doping. The absorption is very low and featureless, indicating that the relaxation rate for reaching equilibrium within the ground state doublet is much faster than the measuring frequency, $\tau^{-1} \gg 10^{5} \mathrm{~s}^{-1}$, and also excluding the occurrence of freezing phenomena of the $\mathrm{Cu}$ or $\mathrm{Nd}$ spins down to $1.5 \mathrm{~K}$.

\section{ACKNOWLEDGMENTS}

This work has been carried out withthin the framework of the CNR-CNPq cooperation project 2001-2002.

${ }^{1}$ W. Henggeler and A. Furrer, J. Phys.: Condens. Matter 10, 2579-2596 (1998).

${ }^{2}$ U. Staub, P. Allenspach, A. Furrer, S.-W. Cheong and Z. Fisk, Solid State Commun. 75, 431 (1990).

${ }^{3}$ A.T. Boothroyd, S.M. Doyle, D.MK. Paul and R. Osborn, Phys. Rev. B 45, 10075 (1992).

${ }^{4}$ W. Henggeler, B. Roessli, A. Furrer, P. Vorderwisch and T. Chatterji, Phys. Rev. Lett. 80, 1300 (1998).

${ }^{5}$ S. Jandl, P. Richard, M. Poirier, V. Nekvasil, A.A. Nugroho, A.A. Menovsky, D.I. Zhigunov, S.N. Barilo and S.V. Shiryaev, Phys. Rev. B 61, 12882 (2000).

${ }^{6}$ N.T. Hien, V.H.M. Duijn, J.H.P. Colpa, J.J.M. Franse and A.A. Menovsky, Phys. Rev. B 57, 5906 (1998).

${ }^{7}$ P. Adelmann, R. Ahrens, G. Czjzek, G. Roth, H. Schmidt and C. Steinleitner, Phys. Rev. B 46, 3619 (1992).

${ }^{8}$ S.J. Collocott, R. Driver and C. Andrikidis, Phys. Rev. B 45, 945 (1992). 
${ }^{9}$ T. Brugger, T. Schreiner, G. Roth, P. Adelmann and G. Czjzek, Phys. Rev. Lett. 71, 2481 (1993).

${ }^{10}$ E. Maiser, W. Mexner, R. Schäfer, T. Schreiner, P. Adelmann, G. Czjzek, J.L. Peng and R.L. Greene, Phys. Rev. B 56, 12961 (1997).

${ }^{11}$ V.D. Fil, G.A. Zvyagina, S.V. Zherlitsyn, I.M. Vitebsky, V.L. Sobolev, S.N. Barilo and D.I. Zhigunov, Mod. Phys. Lett. B 5, 1367 (1991).

12 M. Saint-Paul, J.L. Tholence, S. Piñol, X. Obradors, R.J. Melville and S.B. Palmer, Solid State Commun. 76, 1257 (1990).

13 M. Daturi, M. Ferretti, E.A. Franceschi and M. Minguzzi, Physica C 268, 300 (1996).

${ }^{14}$ M. Matsuda, K. Yamada, K. Kakurai, H. Kadowaki, T.R. Thurston, E. Endoh, Y. Hidaka, R.J. Birgeneau, M.A. Kastner, P.M. Gehring, A.H. Moudden and G. Shirane, Phys. Rev. B 42, 10098 (1990).

15 A.N. Knigavko, H.L. Huang and V.L. Sobolev, J. Appl. Phys. 81, 4154 (1997).

${ }^{16}$ G. Leibfried and N. Breuer, Point Defects in Metals I. (Springer, , 1978).

17 G. Cannelli, R. Cantelli, F. Cordero and F. Trequattrini, Tunneling Sistems in Amorphous and Crystalline Solids. ed. by P. Esquinazi, p. 389 (Springer, Berlin, 1998).

18 A.S. Nowick and B.S. Berry, Anelastic Relaxation in Crystalline Solids. (Academic Press, New York, 1972).

19 M. Hillberg, M.A.C. de Melo, H.H. Klauß, W. Wagener, F.J. Litterst, P. Adelmann and G. Czjzek, Hyperfine Interact. 104, 221 (1997).

${ }^{20}$ G.A. Alers, Physical Acoustics Vol. IV A. ed. by W.P. Mason, p. 277-297 (Academic Press, New York, 1966).

${ }^{21}$ D.C. Johnston, Handbook of Magnetic Materials. ed. by K.H.J. Buschow, p. 1 (North Holland, 1997).

${ }^{22}$ F. Cordero, A. Paolone, R. Cantelli and M. Ferretti, Phys. Rev. B 62, 5309 (2000).

FIG. 1. Elastic energy loss coefficient and relative change of the Young's modulus $E$ of $\mathrm{Nd}_{2-x} \mathrm{Ce}_{x} \mathrm{CuO}_{4}$ samples with $x=0(0.9 \mathrm{kHz}), x=0.05(2 \mathrm{kHz})$ and $x=0.20(1.6 \mathrm{kHz})$.

FIG. 2. Fit of the relative change of the Young's modulus of $\mathrm{Nd}_{2-x} \mathrm{Ce}_{x} \mathrm{CuO}_{4}$ with the paraelastic contribution from a doublet with splitting $\Delta$, Eq. 2 . 


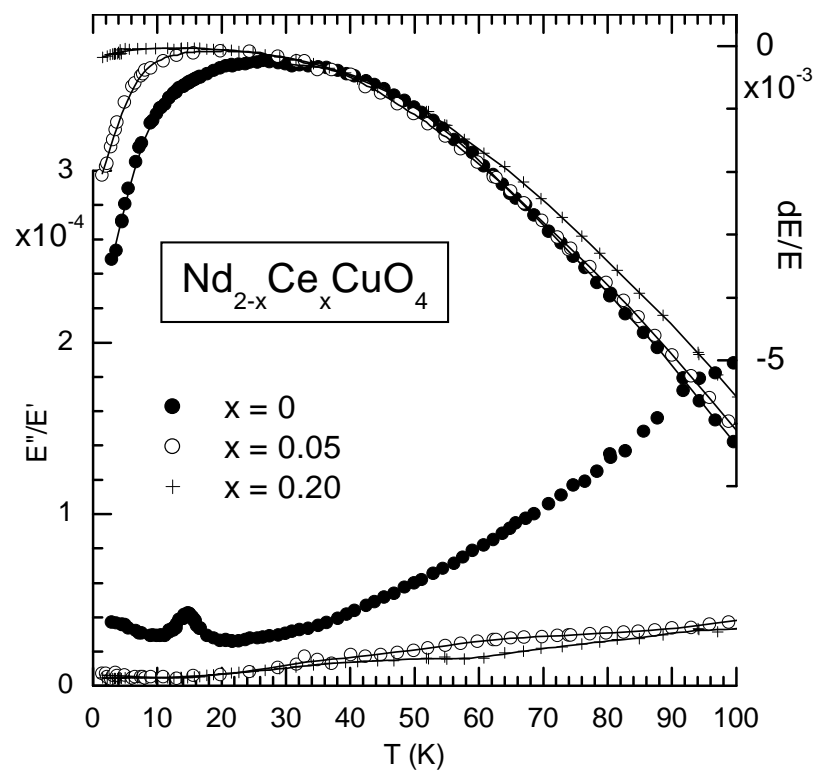




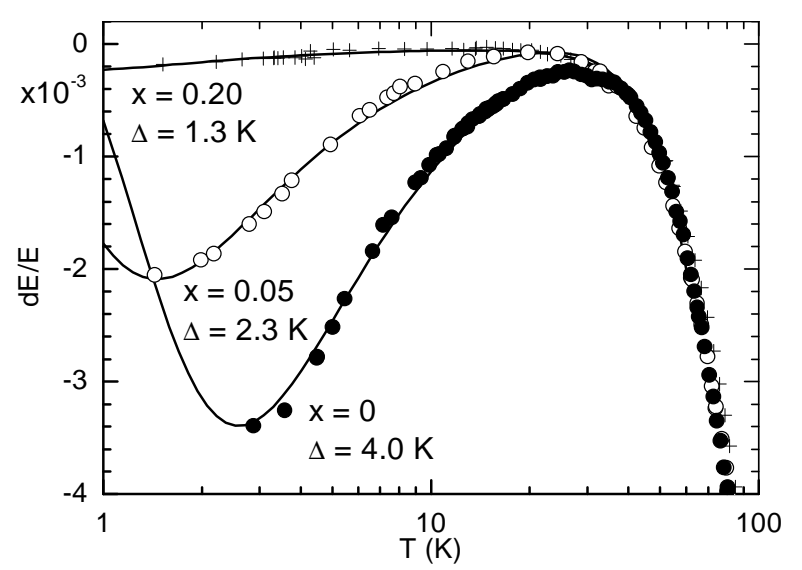

